\title{
Artigo/Article
}

\section{Malária autóctone notificada no Estado de São Paulo: aspectos clínicos e epidemiológicos de 1980 a 2007}

\author{
Autochthonous malaria notified in the State of São Paulo: clinical and epidemiological \\ characteristics from 1980 to 2007
}

\author{
Renata D’Avila Couto ${ }^{1}$, Maria do Rosário Dias de Oliveira Latorre ${ }^{2}$, Silvia Maria Di Santi ${ }^{3}$ e Delsio Natal ${ }^{2}$
}

\begin{abstract}
RESUMO
Introdução: A malária autóctone no Estado de São Paulo caracteriza-se por surtos esporádicos na região oeste e transmissão persistente na região leste onde ocorrem casos oligossintomáticos com baixa parasitemia pelo Plasmodium vivax. Os objetivos deste estudo foram: analisar a completitude das fichas de notificação de malária autóctone; estimar a tendência da incidência de casos autóctones no ESP de 1980 a 2007; analisar o comportamento clínico e epidemiológico dos casos em duas regiões de autoctonia neste período. Métodos: Foi realizado um estudo descritivo com 18 variáveis das FIN de malária do ESP, analisadas em duas regiões e em dois períodos (1980-1993 e 1994-2007). Fontes de dados: SUCEN/SES/SP, SINAN/CVE/SES/ SP e DATASUS. Resultados: A completitude foi superior a $85 \%$ em 11 variáveis. A tendência da incidência foi decrescente. Foram notificados 821 casos autóctones, 91,6\% na região leste, predominando Plasmodium vivax. A infecção assintomática teve maior porcentagem no segundo período $(p<0,001)$. Conclusões: A completitude das informações foi considerada satisfatória. As diferenças clínicas encontradas merecem atenção da vigilância epidemiológica que deve lidar com o desafio da infecção assintomática por Plasmodium.
\end{abstract}

Palavras-chaves: Malária. Epidemiologia. Vigilância epidemiológica.

\begin{abstract}
Introduction: Autochthonous malaria in the State of São Paulo is characterized by sporadic outbreaks in the western region and by persistent transmission in the eastern region, where oligosymptomatic cases with low parasitemia due to Plasmodium vivax occur. The objectives of this study were to assess the completeness of autochthonous malaria notification forms; to estimate the incidence trends of autochthonous cases in the State of São Paulo from 1980 to 2007; and to analyze the clinical and epidemiological patterns of cases in two autochthonous regions over this period. Methods: This was a descriptive study that analyzed 18 variables on the notification form for malaria in the State of São Paulo, comparing these two regions over two periods (1980-1993 and 1994-2007). The data sources were SUCEN/SES/SP, SINAN/CVE/ SES/SP and DATASUS. Results: The completeness was greater than $85 \%$ for 11 variables. The incidence trend was decreasing. There were 821 autochthonous cases: $91.6 \%$ occurred in the eastern region, predominantly due to Plasmodium vivax. Asymptomatic infection had a higher percentage in the second period $(\mathrm{p}<0.001)$. Conclusions: The completeness of the information was satisfactory. The clinical differences observed deserve attention from epidemiological surveillance agencies, which need to deal with the challenge of asymptomatic infection by Plasmodium.
\end{abstract}

Key-words: Malaria. Epidemiology. Epidemiological surveillance.

1. Curso de Pós Graduação pela Faculdade de Saúde Pública, Universidade de São Paulo, São Paulo, SP. 2. Departamento de Epidemiologia, Faculdade de Saúde Pública, Universidade de São Paulo, São Paulo, SP. 3. Superintendência de Controle de Endemias do Estado de São Paulo, São Paulo, SP.

Endereço para correspondência: Dra Renata D’Avila Couto. Depto de Epidemiologia/FSP/USP. Av. Dr. Arnaldo 715, Cerqueira César, 01246-904 São Paulo, SP.

Tel: 5511 3061-7710/3061-7737

e-mail: renatadavilacouto@yahoo.com.br

Recebido para publicação em 11/03/2009

Aceito em 11/01/2010
INTRODUÇÃO

A malária humana é uma doença infecciosa cujos agentes etiológicos são protozoários do gênero Plasmodium. A transmissão ocorre de forma natural por mosquitos vetores do gênero Anopheles ou de forma induzida, por transfusão de sangue ou compartilhamento de agulhas e seringas infectadas com plasmódios ${ }^{1,2,3}$. Na década de 70 , em consequência dos programas de erradicação mundial, $53 \%$ da população residente em áreas malarígenas ficaram livres do risco da doença. Entretanto, a situação se deteriorou na década de 80 e ocorreu aumento progressivo do número de casos no Brasil, com registro de 169.871 casos novos. Diante do recrudescimento da malária, houve uma revisão da estratégia global de erradicação com adoção de abordagens mais específicas diante do caráter focal da doença e suas situações peculiares ${ }^{1,4}$. Na década de 90, a política de assentamento rural e extrativismo na região Amazônica proporcionaram um novo aumento do número de casos no país, chegando a 637.474 casos em $1999^{5}$. De 2000 a 2007, o número de casos variou de 615.247 a $458.624^{5,6}$.

O Estado de São Paulo refletiu a situação de recrudescimento da malária na década de $80 \mathrm{com}$ aumento dos casos importados e autóctones. Houve registro anual médio de 40 casos de autoctonia entre 1983 e $1993^{7,8}$. Na década atual, este estado vem apresentando aumento do número absoluto de casos autóctones, com notificação de 57 casos em 2006, representando $42 \%$ dos casos autóctones notificados na região extra-amazônica naquele ano ${ }^{6,7}$.

A autoctonia da malária em São Paulo vem sendo descrita em duas regiões geográficas distintas ${ }^{8-11}$. A primeira é a região da Serra do Mar, ecossistema com presença da Mata Atlântica, onde anofelinos vetores do subgênero Kerteszia podem ser encontrados em alta densidade. A segunda é a região oeste do estado, especialmente nas áreas das bacias hidrográficas dos rios Paraná, Paranapanema e São José dos Dourados. Nesta área, a presença de anofelinos vetores do 
subgênero Nyssorhynchus, aliada ao fluxo de portadores da doença provenientes da Região Amazônica, foi local de atenção para a vigilância epidemiológica nas décadas de 80 e 90.

A vigilância epidemiológica da malária no Estado de São Paulo foi centralizada e de competência da Superintendência de Controle de Endemias da Secretaria de Estado da Saúde de São Paulo (SUCENSES/SP) de 1975 até 2003, quando se iniciou a descentralização da vigilância deste agravo. A SUCEN é estruturada em dez Serviços Regionais (SR) e conta, atualmente, com o apoio de 14 unidades hospitalares de referência em diferentes regiões do estado. As condutas de controle de foco são hoje realizadas mediante parceria das esferas municipais com a SUCEN e incluem: reconhecimento e delimitação da área de foco, identificação de vetor e busca ativa de casos com coleta de sangue para exame laboratorial daquelas pessoas que apresentem sintomas da doença na área do foco identificado ${ }^{9}$. A partir de 2003, a notificação de malária no estado passou a ser pelo Sistema de Informação de Agravos de Notificação (SINAN).

No Estado de São Paulo, vários focos de malária autóctone foram investigados e descritos na literatura, tanto na região de Mata Atlântica quanto no Planalto, principalmente nas décadas de 80 e de 90 . A infecção é descrita como assintomática ou oligossintomática, com baixa parasitemia do principal agente, o Plasmodium vivax. A coleta sistemática de dados de investigação passou a ser realizada utilizando-se fichas de notificação de casos, as quais foram sendo modificadas ao longo dos anos. Estas fichas contem variáveis que refletem aspectos importantes da situação clínica, epidemiológica e de controle da malária autóctone.

O presente estudo tem os objetivos de verificar a completitude das fichas de notificação de malária autóctone, estimar a tendência da série histórica de casos de malária autóctone notificados no Estado de São Paulo de 1980 a 2007 e analisar mudanças no comportamento clínico e epidemiológico dos casos em dois períodos (de 1980 a 1993 e de 1994 a 2007) nas duas regiões distintas de autoctonia: a região leste, sob influência da Mata Atlântica, e a região oeste no planalto.

\section{MÉTODOS}

Foi realizado um estudo epidemiológico descritivo. Foram analisadas as fichas de notificação (FIN) de malária dos casos confirmados autóctones no Estado de São Paulo, de janeiro de 1980 a dezembro de 2007. A definição de autoctonia foi aquele caso de infecção por plasmódio cujo local provável de infecção foi no Estado de São Paulo. De 1980 a 2003, foram levantados os dados das FIN utilizadas pela SUCEN. A partir de 2003, os dados foram levantados do Sistema de Informações de Agravos de Notificação (SINAN). Dado que a SUCEN manteve um banco paralelo de notificação durante a fase de transição para o novo instrumento de notificação entre 2003 e 2007, optou-se por conferir os dados de ambas as fontes a fim de se obter dados fidedignos.

Ao longo do período de estudo, foram utilizadas cinco diferentes FIN de malária: três da SUCEN (1980-1984; 1985-1989; 1990 a 2002) e duas do SINAN (SINAN Windows, de 2003 a 2005, e a atual do SINAN NET). Foi construído um banco de dados com 18 variáveis de interesse coletadas destes instrumentos de notificação. Ao longo do tempo, algumas destas variáveis foram mantidas, outras acrescentadas ou retiradas. Aquelas variáveis que, em determinado período, não existiam na FIN, foram denominadas, neste estudo, variáveis não aplicáveis ou sem aplicabilidade.

A completitude foi calculada pela porcentagem de preenchimento das seguintes variáveis: idade, gênero, ocupação, estado gestacional, data da notificação, data do início dos sintomas, data de tratamento, sinaise sintomas, espécie de plasmódio, parasitemia, evolução clínica (óbito ou não), destino do paciente (hospital ou ambulatório), tipo de busca (ativa preventiva, passiva ou controle de foco), município de infecção, doação e transfusões sanguíneas prévias, uso de drogas endovenosas e infecções prévias por plasmódio. Foi calculada a oportunidade de tratamento, correspondendo ao intervalo entre a data de tratamento e a data do início dos sintomas, em dias.

A comparação dos aspectos clínicos e epidemiológicos foi realizada com variáveis cuja perda total de informação foi inferior a 15\% em todo o período estudado. Perda total de informação foi calculada pela soma da porcentagem de falta de preenchimento e da porcentagem de não aplicabilidade da variável. Considerou-se região leste do Estado de São Paulo a área influenciada pela Mata Atlântica, correspondente aos 180 municípios de abrangência dos Serviços Regionais (SR) da SUCEN da Grande São Paulo (1), São Vicente (2), Taubaté (3) e Sorocaba (4), como mostra a Figura 1. Já a região oeste, ou planalto, corresponde aos demais 465 municípios de abrangência dos SR de Campinas (5), Ribeirão Preto (6), São José do Rio Preto (7), Araçatuba (9), Presidente Prudente (10) e Marília (11) - Figura 1. A mesma comparação de aspectos clínicos e epidemiológicos foi feita em dois períodos: 1980 a 1993 e 1994 a 2007. Justifica-se este feito porque o número de casos de malária importada era maior no primeiro período, podendo ter maior influência na introdução de casos na região oeste do estado. Além disso, em 1988 foi criado o Sistema Único de Saúde (SUS), possibilitando mudanças em assistência e diagnóstico, principalmente após alguns anos de sua implantação.

Para o cálculo da taxa de incidência anual de malária autóctone, dividiu-se o número de casos autóctones no estado por ano pela população anual estimada do estado para este território, utilizando-se dados populacionais do Departamento de Informática do Sistema Único de Saúde (DATASUS). A taxa de incidência anual foi calculada para a região leste, tendo como numerador os casos autóctones por ano ocorridos nos SR 1, 2, 3 e 4 e o denominador a população anual dos municípios de abrangência destes SR. Na região oeste o numerador foi o número anual de casos ocorridos nos SR 5, 6, 8, 9, 10 e 11 e o denominador a população dos municípios de abrangência destes SR, segundo as mesmas fontes citadas anteriormente.

A estimativa da tendência das taxas de incidência de casos autóctones foi calculada utilizando-se um modelo de regressão polinomial de primeiro grau. As taxas de incidência foram consideradas como variáveis dependentes (Y) e os anos-calendário de estudo como variável independente $(\mathrm{X})$. Para evitar a autocorrelação entre os termos da equação de regressão, a variável ano foi transformada em variável ano-centralizada (X menos o ponto médio da série histórica) ${ }^{12}$. Optouse pelo alisamento da série história utilizando-se a média móvel de três anos. Foi feita a análise de resíduos, observada a suposição de homocedasticidade e aderência à distribuição normal.

As análises descritivas do banco de dados, a estimativa de tendência da série histórica e as análises das variáveis qualitativas pelo teste estatístico do qui-quadrado, foram feitas no programa EPI-INFO para Windows, versão 3.4.0. Foi considerado o nível de significância de $5 \%$.

\section{Ética}

A pesquisa foi feita a partir de dados secundários individuais da SUCEN e SINAN, sendo divulgados somente dados agrupados. O protocolo de pesquisa foi submetido e aprovado pelo Comitê de Ética da Faculdade de Saúde Pública da Universidade de São Paulo e pela SUCEN. 


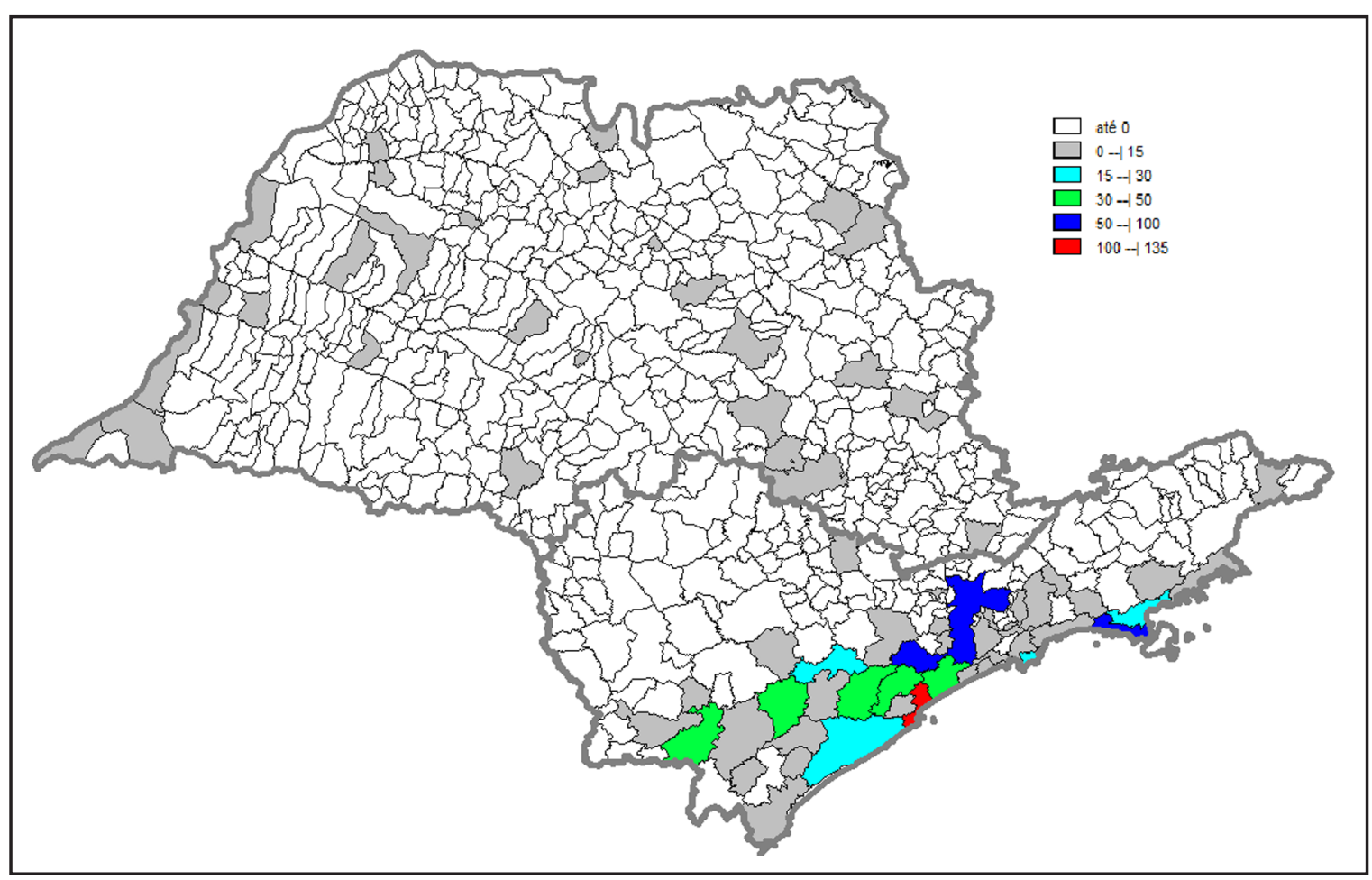

FIGURA 1 - Número de casos (n⿳o 816) de malária autóctone segundo municípios e regiões leste e oeste do Estado de São Paulo, 1980 a 2007.

\section{RESULTADOS}

No Estado de São Paulo, houve registro de 821 casos de autoctonia de 1980 a 2007, sendo que 91,6\% deles ocorreram na região leste (Figura 1). Os municípios com maior número de casos nesta região foram Peruíbe: 135 casos, Juquitiba: 81, São Paulo: 65, São Sebastião: 58, Miracatu: 44, Iporanga: 36, Pedro de Toledo: 36 e Sete Barras: 35. Na região oeste, destacaram-se Araçatuba: 14, Palmeira d'Oeste: 9, Presidente Epitácio: 6, Teodoro Sampaio: 5 e Castilho: 5. A doença teve predomínio no sexo masculino (72,1\%). A média de idade dos casos foi de 32 anos e mediana de 31 anos, sendo a faixa etária de 20 a 39 anos a mais acometida (39\%). O agente etiológico mais frequente foi Plasmodium vivax, causando 97,2\% das infecções. Outras espécies representaram 2,8\%: 14 casos de Plasmodium falciparum; cinco casos de Plasmodium malariae; três casos de infecção mista (Plasmodium vivax e Plasmodium falciparum) e um registro de Plasmodium ovale. Em relação à sintomatologia, 9,6\% dos casos foram assintomáticos. Os sintomas mais frequentes foram: febre $(85,3 \%)$, cefaléia $(72,1 \%)$ e calafrios $(59,7 \%)$. Transfusão sanguínea prévia não foi comparada por região por ter ocorrido em $0,4 \%$ dos casos. A oportunidade de tratamento ocorreu em 12,6\% até o terceiro dia do início dos sintomas, 33,9\% entre 4 e 9 dias, $43 \%$ entre 10 e 30 dias e $10,5 \%$ com mais de 30 dias.

Os resultados em relação à completitude das variáveis estão apresentados na Tabela 1. Das 18 variáveis avaliadas, 7 (38,9\%) apresentaram perda de informação total maior que $15 \%$. Entre estas, o tipo de busca da infecção ocorreu de forma passiva em $58,6 \%$ dos casos, $30,8 \%$ como controle de foco e $10,6 \%$ por busca ativa. A parasitemia teve a seguinte distribuição: $<1 / 2+$ em $11,8 \%$ dos casos, $1 / 2+$ em $49,2 \%$, + em 19,7\%, ++ em 17,5\% e +++ /++++ em 1,8\%. Os pacientes foram acompanhados ambulatorialmente em $86,5 \%$ dos casos. Não houve registro de óbitos em casos autóctones. Em
TABELA 1 - Completitude das variáveis selecionadas das fichas de notificação de malária segundo aplicabilidade, ausência de preenchimento e perda total. Malária autóctone no Estado de São Paulo, 1980 a 2007.

\begin{tabular}{|c|c|c|c|c|c|c|}
\hline \multirow[b]{2}{*}{ Variável } & \multicolumn{2}{|c|}{ Não aplicável } & \multicolumn{2}{|c|}{ Sem preenchimento } & \multicolumn{2}{|c|}{ Perda total } \\
\hline & $\mathrm{n}^{\mathrm{o}}$ & $\%$ & $\mathrm{n}^{\mathrm{o}}$ & $\%$ & $\mathrm{n}^{\circ}$ & $\%$ \\
\hline Idade & 0 & 0,0 & 2 & 0,2 & 2 & 0,2 \\
\hline Sexo & 0 & 0,0 & 0 & 0,0 & 0 & 0,0 \\
\hline Gestação & 129 & 56,0 & 0 & 0,0 & 129 & 56,0 \\
\hline Ocupação & 0 & 0,0 & 126 & 15,0 & 126 & 15,0 \\
\hline Data da notificação & 0 & 0,0 & 0 & 0,0 & 0 & 0,0 \\
\hline Data dos primeiros sintomas & 0 & 0,0 & 38 & 4,6 & 38 & 4,6 \\
\hline Data do tratamento & 0 & 0,0 & 114 & 13,9 & 114 & 13,9 \\
\hline Sinais e sintomas & 58 & 7,0 & 9 & 1,1 & 67 & 8,1 \\
\hline Espécies de plasmódio & 0 & 0,0 & 10 & 1,2 & 10 & 1,2 \\
\hline Parasitemia & 148 & 18,0 & 79 & 9,6 & 227 & 27,6 \\
\hline Evolução & 341 & 41,5 & 9 & 1,1 & 350 & 42,6 \\
\hline Município de infecção & 0 & 0,0 & 3 & 0,4 & 3 & 0,4 \\
\hline Destino do paciente & 205 & 25,0 & 30 & 3,6 & 235 & 28,6 \\
\hline Tipo de busca & 255 & 31.1 & 10 & 1,2 & 265 & 32,3 \\
\hline Episódio de malária anterior & 58 & 7,1 & 16 & 1,9 & 74 & 9,0 \\
\hline Transfusão sanguinea prévia & 59 & 7,2 & 46 & 5,6 & 105 & 12,8 \\
\hline Doação sanguinea prévia & 329 & 40,0 & 42 & 5,2 & 371 & 45,2 \\
\hline Uso de drogas endovenosas & 401 & 48,8 & 100 & 12,2 & 501 & 61,0 \\
\hline
\end{tabular}

Fontes: SINAN/SES/SP e SUCEN/SES/SP.

relação ao uso de drogas endovenosas, 99,7\% negaram o hábito. Há registro de $6(1,3 \%)$ casos de doação sanguínea prévia.

A distribuição dos casos segundo região de autoctonia está descrita na Tabela 2. Comparando-se as regiões leste e oeste, não houve diferenças na distribuição dos casos em relação ao sexo, faixa etária, ocupação, oportunidade de tratamento e infecção prévia por plasmódio. A região oeste apresentou menor porcentagem de casos 
TABELA 2 - Distribuição dos casos de malária autóctone segundo variáveis* selecionadas das fichas de notificação, por regiões leste e oeste do estado de São Paulo, 1980 a 2007.

\begin{tabular}{|c|c|c|c|c|c|c|c|c|c|c|c|c|c|c|c|c|c|}
\hline \multirow[b]{2}{*}{ Variável } & \multicolumn{2}{|c|}{ Região leste } & \multicolumn{2}{|c|}{ Região oeste } & \multicolumn{2}{|c|}{ Total } & \multirow[b]{2}{*}{$\mathrm{X}^{2}$ yates } & \multirow[b]{2}{*}{$\mathrm{p}$} & \multirow[b]{2}{*}{ Variável } & \multicolumn{2}{|c|}{ Região leste } & \multicolumn{2}{|c|}{ Região oeste } & \multicolumn{2}{|c|}{ Total } & \multirow[b]{2}{*}{$\mathrm{X}^{2}$ yates } & \multirow[b]{2}{*}{$\mathrm{p}$} \\
\hline & $\mathrm{n}^{\mathrm{o}}$ & $\%$ & $\mathrm{n}^{\mathrm{o}}$ & $\%$ & $\mathrm{n}^{\mathrm{o}}$ & $\%$ & & & & $\mathrm{n}^{\mathrm{o}}$ & $\%$ & $\mathrm{n}^{\mathrm{o}}$ & $\%$ & $\mathrm{n}^{\mathrm{o}}$ & $\%$ & & \\
\hline Sexo & & & & & & & & & Náuseas & & & & & & & & \\
\hline masculino & 536 & 71,3 & 56 & 81,2 & 592 & 72,1 & 2,6 & 0,107 & $\operatorname{sim}$ & 140 & 20,2 & 11 & 7,3 & 151 & 20,0 & 0,03 & 0,862 \\
\hline feminino & 216 & 28,7 & 13 & 18,8 & 229 & 27,9 & & & não & 554 & 79,8 & 49 & 81,7 & 603 & 80,0 & & \\
\hline Faixa etária (anos) & & & & & & & & & Vômito & & & & & & & & \\
\hline 0 a 19 & 200 & 26,7 & 19 & 27,5 & 219 & 26,7 & 0,86 & 0,930 & $\operatorname{sim}$ & 136 & 19,6 & 30 & 50,0 & 166 & 22,0 & $27,99<$ & $0,001^{*}$ \\
\hline 20 a 29 & 149 & 19,9 & 12 & 17,4 & 161 & 19,7 & & & não & 558 & 80,4 & 30 & 50,0 & 588 & 78,0 & & \\
\hline 30 a 39 & 143 & 19,1 & 15 & 21,7 & 158 & 19,3 & & & Diarréia & & & & & & & & \\
\hline 40 a 49 & 128 & 17,1 & 13 & 18,8 & 141 & 17,2 & & & $\operatorname{sim}$ & 47 & 6,8 & 5 & 8,3 & 52 & 6,9 & 0,04 & 0,848 \\
\hline$>50$ & 130 & 17,3 & 10 & 14,5 & 140 & 17,1 & & & não & 647 & 93,2 & 55 & 91,7 & 702 & 93,1 & & \\
\hline Infecção assintomátic & & & & & & & & & Inapetência & & & & & & & & \\
\hline $\operatorname{sim}$ & 77 & 10,4 & 1 & 1,5 & 78 & 9,6 & 4,69 & $0,030^{*}$ & $\operatorname{sim}$ & 144 & 20,7 & 27 & 45,0 & 171 & 22,7 & $17,16<$ & $0,001^{*}$ \\
\hline não & 666 & 89,6 & 67 & 98,5 & 733 & 90,4 & & & não & 550 & 79,3 & 33 & 55,0 & 583 & 77,3 & & \\
\hline Febre & & & & & & & & & Mal estar & & & & & & & & \\
\hline $\operatorname{sim}$ & 583 & 84,0 & 60 & 100,0 & 643 & 85,3 & 10,02 & $0,001^{*}$ & $\operatorname{sim}$ & 162 & 23,3 & 24 & 40,0 & 186 & 24,7 & $7,37<$ & $0,007^{*}$ \\
\hline não & 111 & 16,0 & 0 & 0,0 & 111 & 14,7 & & & não & 532 & 76,7 & 36 & 60,0 & 568 & 75,3 & & \\
\hline Calafrio & & & & & & & & & Dor na nuca & & & & & & & & \\
\hline $\operatorname{sim}$ & 396 & 57,1 & 54 & 90,0 & 450 & 59,7 & $23,55<1$ & $0,001^{*}$ & $\operatorname{sim}$ & 131 & 18,9 & 21 & 35,0 & 152 & 20,2 & $7,95<$ & $0,005^{*}$ \\
\hline não & 298 & 42,9 & 6 & 10,0 & 304 & 40,3 & & & não & 563 & 81,1 & 39 & 65,0 & 602 & 79,8 & & \\
\hline Cefaléia & & & & & & & & & Espécie de plasmódio & & & & & & & & \\
\hline $\operatorname{sim}$ & 494 & 71,2 & 50 & 83,3 & 544 & 72,1 & 3,48 & 0,062 & Plasmodium vivax & 733 & 98,7 & 55 & 80,9 & 788 & 97,2 & $65,10<$ & $0,001^{*}$ \\
\hline não & 200 & 28,8 & 10 & 16,7 & 210 & 27,9 & & & outros & 10 & 1,3 & 13 & 19,1 & 23 & 2,8 & & \\
\hline Lombalgia & & & & & & & & & Oportunidade de & & & & & & & & \\
\hline $\operatorname{sim}$ & 147 & 21,2 & 21 & 35,0 & 168 & 22,3 & 5,32 & $0,021^{*}$ & tratamento (dias) & & & & & & & & \\
\hline não & 547 & 78,8 & 39 & 65,0 & 586 & 77,7 & & & 0 & 13 & 1,9 & 1 & 1,5 & 14 & 1,8 & 1,84 & 0,766 \\
\hline Artralgia & & & & & & & & & $>30$ & 74 & 10,7 & 5 & 7,5 & 79 & 10,4 & & \\
\hline $\operatorname{sim}$ & 135 & 19,5 & 14 & 23,3 & 149 & 19,8 & 0,31 & 0,579 & Ocupação & & & & & & & & \\
\hline não & 559 & 80,5 & 46 & 76,7 & 605 & 80,2 & & & estudante & 120 & 18,2 & 11 & 32,4 & 131 & 18,8 & 8,07 & 0,089 \\
\hline Dor muscular & & & & & & & & & lavrador & 121 & 18,3 & 8 & 23,5 & 129 & 18,6 & & \\
\hline $\operatorname{sim}$ & 235 & 33,3 & 26 & 43,3 & 261 & 34,6 & 1,79 & 0,181 & lazer & 107 & 16,2 & 1 & 2,9 & 108 & 15,5 & & \\
\hline não & 459 & 66,1 & 34 & 56,7 & 493 & 65,4 & & & caseiro & 92 & 13,9 & 5 & 14,7 & 97 & 14,0 & & \\
\hline Dor abdominal & & & & & & & & & outros & 221 & 33,4 & 9 & 26,5 & 230 & 33,1 & & \\
\hline $\operatorname{sim}$ & 110 & 15,9 & 11 & 9,1 & 121 & 16,0 & 0,1 & 0,749 & & & & & & & & & \\
\hline não & 584 & 84,1 & 49 & 7,7 & 633 & 84,0 & & & & & & & & & & & \\
\hline
\end{tabular}

${ }^{*} \mathrm{n}^{\mathrm{o}}$ das variáveis conforme suas completitudes.

Fontes: SINAN/SES/SP e SUCEN/SES/SP.

assintomáticos $(\mathrm{p}=0,030)$ e maior proporção de febre, cefaléia, calafrio, vômitos, inapetência, mal estar e dor na nuca $(\mathrm{p}<0,05)$. Além disso, outras espécies de Plasmodium, diferentes de Plasmodium vivax, ocorreram mais nesta região $(\mathrm{p}<0,001)$.

Na comparação entre os períodos (Tabela 3), observou-se diminuição da ocorrência de casos na faixa etária de zero a 19 anos e aumento na faixa de 30 a 59 anos no segundo período $(p=0,042)$. Em relação à ocupação, houve diminuição da porcentagem de casos em estudantes e lavradores, e aumento da proporção da ocupação lazer $(\mathrm{p}<0,001)$. Além disso, a infecção assintomática foi mais frequente no segundo período $(\mathrm{p}<0,001)$. Analisando somente os sintomas da infecção por Plasmodium vivax, as proporções de febre, cefaléia e vômitos foram menores no segundo período, enquanto sintomas mais inespecíficos, como lombalgia, artralgia, inapetência e mal estar foram mais frequentes $(p<0,05)$.

A estimativa da tendência das taxas de incidência de malária autóctone no Estado de São Paulo de 1980 a 2007 mostrou-se decrescente (Figura 2). Tanto o Estado de São Paulo em geral, quanto às regiões leste e oeste, analisadas separadamente, apresentaram tendências decrescentes, com modelos polinomiais significativos. Os modelos finais utilizados foram: para o Estado de São Paulo $\mathrm{Y}=0,085-0,044 \mathrm{X}\left(\mathrm{r}^{2}=0,32 ; \mathrm{p}=0,003\right)$; para a região leste: $\mathrm{Y}=0,152$ $0,004 X\left(r^{2}=0,16 ; p=0,044\right)$ e para a região oeste: $Y=0,016-0,001 X$ $\left(\mathrm{r}^{2}=0,22 ; \mathrm{p}=0,016\right)$. 
TABELA 3 - Distribuição dos casos de malária autóctone segundo variáveis* selecionadas das fichas de notificação, em dois períodos no estado de São Paulo de 1980 a 2007.

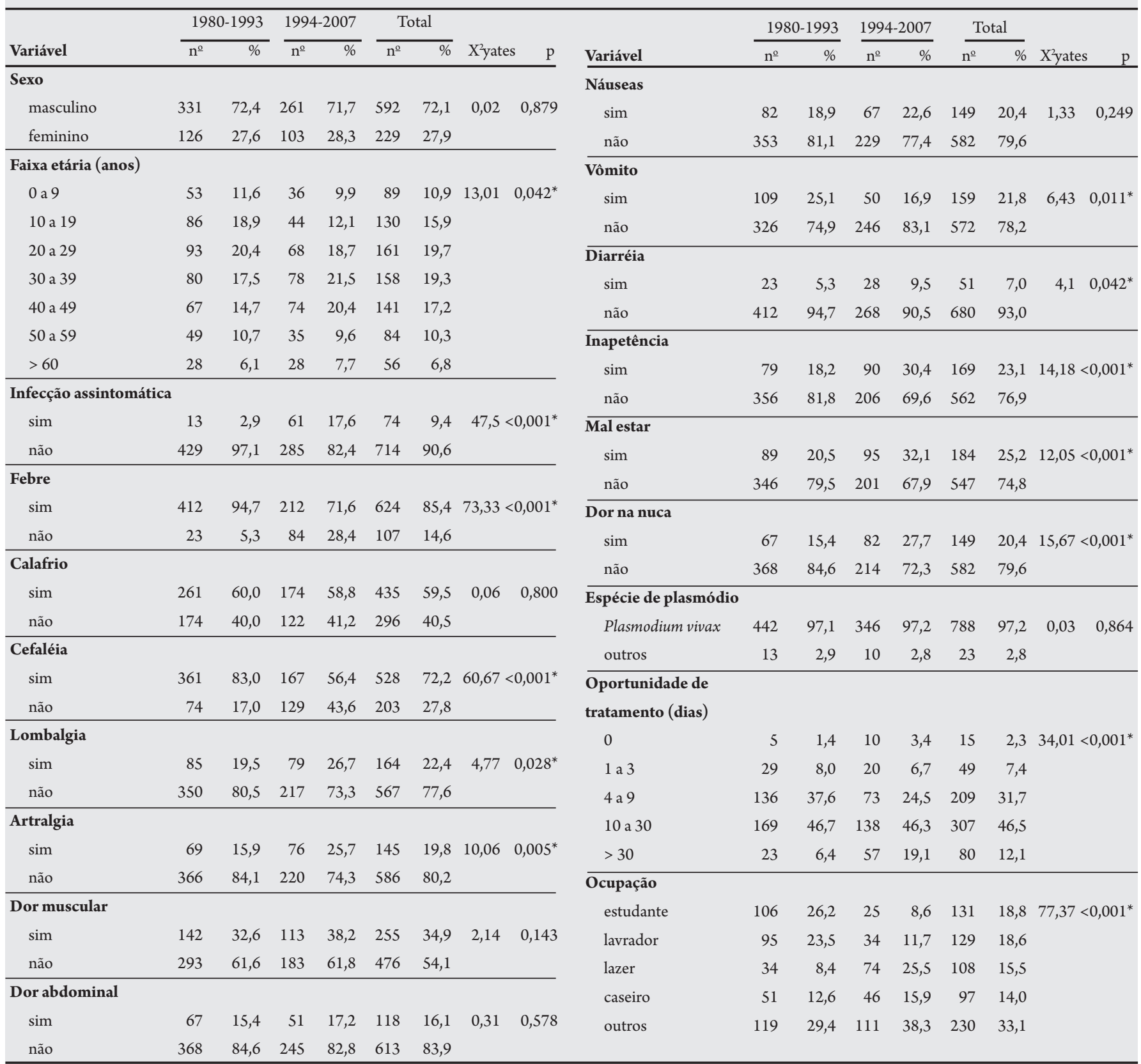

* $n^{\circ}$ das variáveis conforme suas completitudes

Fontes: SINAN/SES/SP e SUCEN/SES/SP

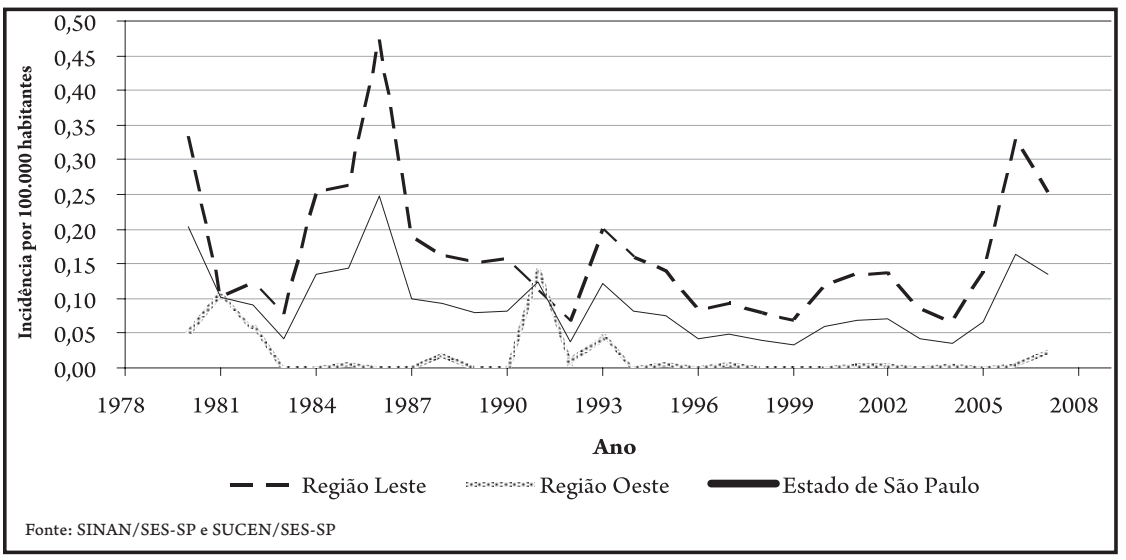

FIGURA 2 - Taxa de incidência de malária autóctone no Estado de São Paulo, segundo região leste e oeste, 1980 a 2007. 


\section{DISCUSSÃO}

Estudos realizados com bancos de dados secundários são importantes para se avaliar e aprimorar os bancos de informações usados nos sistemas de vigilância. São conhecidas as limitações encontradas na utilização dessas fontes ${ }^{13}$, como a completitude das variáveis, erros de digitação e inconsistências de dados. Como exemplos neste estudo têm-se: a notificação de Plasmodium ovale, possível erro de digitação; duplicidade de dados, as quais foram corrigidas; e a completitude dos dados, mostrada na Tabela 1. Apesar das limitações descritas, o objetivo final da coleta de dados por meio das fichas de notificação de agravos é analisá-los e utilizálos para fins de políticas públicas e ações de prevenção e controle. $\mathrm{O}$ aprimoramento da coleta e dos bancos de dados se faz fundamental para tal objetivo.

Em relação à completitude, das 18 variáveis analisadas neste estudo, 7 (39\%) apresentavam perda total de informação maior do que $15 \%$. Porém, a perda total de informação encontrada ocorreu principalmente por não aplicabilidade da variável ao longo de toda a série histórica. Neste grupo, destacam-se as variáveis: doação sanguínea, com $40 \%$ de não aplicabilidade, ausente nas fichas de 1980 a 1984 e 2007; gestação e evolução, não aplicáveis de 1980 a 1989; sinais e sintomas, uma variável não mais aplicável a partir de 2007. Frente ao fato de que apenas $18 \%$ das infecções notificadas de 1994 a 2007 foram assintomáticas, pode-se discutir a relevância da caracterização dos sinais e sintomas dos pacientes infectados para a vigilância epidemiológica e considerar sua re-incorporação na FIN do SINAN. O mesmo poderia ser feito com a variável doação sanguínea, suprimida também a partir de 2007 e de grande importância quando se considera o desafio da infecção assintomática e a possibilidade real de malária por via transfusional a partir destes $\operatorname{casos}^{14,15}$. Já a variável data de tratamento apresentou perda de informação decorrente da falta de preenchimento em 13,8\% das FIN. Esta variável é fundamental para se avaliar a oportunidade de tratamento, cuja precocidade é uma das prioridades do Plano Nacional de Prevenção e Controle de Malária ${ }^{5}$; portanto, o aprimoramento do preenchimento desta variável se faz necessário.

Alguns estudos descreveram somente a malária autóctone no Estado de São Paulo. A maior parte trata-se de relatos bem descritos de focos de autoctonia nas décadas de 80 e 90 $0^{16-20}$. Em 2004, foi publicado um estudo sobre os aspectos da vigilância epidemiológica de todos os casos de malária no Estado de São Paulo no período de 1983 a $2003^{7}$, incluindo considerações sobre a malária autóctone. O presente estudo foi restrito aos casos autóctones de 1980 a 2007, sendo que os aspectos epidemiológicos da autoctonia encontrados foram semelhantes ao descrito para o estado, como a tendência decrescente das taxas de incidência e o perfil do indivíduo acometido, predominantemente do sexo masculino e com faixa etária economicamente ativa. A infecção autóctone por Plasmodium vivax vem sendo descrita como assintomática ou oligossintomática, com baixa parasitemia ${ }^{5,7,10}$. Constatamos, porém, que apenas $9,6 \%$ dos casos foram assintomáticos, tendo sido os sintomas mais frequentes: febre $(85,3 \%)$, cefaléia $(72,1 \%)$ e calafrios $(59,7 \%)$. A baixa parasitemia também foi confirmada neste estudo.

Analisando as diferenças entre as duas regiões distintas de autoctonia, observou-se que a região oeste apresentou maior número de infecção sintomática com maior proporção de febre, calafrio, vômitos, inapetência, mal estar e dor na nuca. Isto pode ser decorrente da maior presença nesta região de outros agentes etiológicos diferentes do Plasmodium vivax, principalmente o Plasmodium falciparum, que geralmente leva a um quadro mais sintomático. O principal surto notificado por este agente ocorreu em Palmeira D’Oeste, região de São José do Rio Preto, com 9 casos notificados em 1981, a partir de dois casos importados. Em 1986, foi publicado outro surto de malária por este agente no município de Panorama, também no extremo oeste do estado, com 10 casos em $1984^{17}$. Neste surto, não foi possível detectar a fonte introdutora da infecção. Estes casos não constam no banco analisado neste estudo, uma vez que estas FIN não foram encontradas no arquivo morto da SUCEN. Em Campinas foi descrito um caso por este agente em $1983^{16}$. Também nesta região de planalto não foi detectado o caso introduzido, mas vetores Anopheles (Nyssorhynchus) albitarsis foram encontrados ${ }^{21}$.

No segundo período da série histórica, um resultado relevante foi a mudança das faixas etárias acometidas, diminuindo o número de casos na faixa etária de 0 a 19 anos e aumentando na de 30 a 59 anos. Da mesma forma, também houve mudança na distribuição da ocupação dos casos notificados, com diminuição da porcentagem de estudantes e lavradores e aumento da proporção da ocupação descrita como lazer. Isto já havia sido apontado por outros autores, refletindo tendências de aumento de práticas de ecoturismo e lazer na região de Serra do $\mathrm{Mar}^{7,10}$. Porém, esses achados merecem algumas considerações: a perda de informação foi maior no segundo período comparado ao primeiro e não houve padronização no preenchimento do campo ocupação. Este por vezes foi preenchido como o motivo do deslocamento para a área de exposição à doença, sendo então preenchido como lazer. Atualmente, este campo tem uma definição mais estrita quanto ao seu preenchimento na FIN do SINAN.

A ocorrência de infecção assintomática também teve maior porcentagem neste segundo período. Comparando-se os sintomas da infecção por Plasmodium vivax, as proporções de febre, cefaléia e vômitos diminuíram. Por outro lado, sintomas mais inespecíficos como lombalgia, artralgia, inapetência e mal estar aumentaram. É difícil afirmar os motivos desta aparente mudança. Uma das hipóteses é que esses casos estariam sendo diagnosticados mais precocemente, por busca ativa ou de controle de foco; porém, ao se analisar o tipo de busca em casos assintomáticos, esta informação ficou prejudicada porque estava ausente em $60 \%$ das notificações. Outra hipótese seria a mudança no método diagnóstico, com o uso de biologia molecular na detecção precoce do plasmódio. Este estudo, porém, só diz respeito aos casos confirmados por hemoscopia. Por fim, a baixa parasitemia encontrada pode contribuir para a infecção assintomática.

A estimativa da tendência das taxas de incidência confirmou-se decrescente no estado. Porém, houve aumento do número absoluto de casos autóctones confirmados a partir de 2005, com 57 casos notificados em $2006^{5,6}$, e isto foi refletido na análise de tendência. Fazse aconselhável o acompanhamento da tendência da incidência, uma vez que os três últimos anos da série estudada apresentaram um pico que pode refletir um novo aumento da incidência, como ocorrido entre 1983 e 1987, ou voltar ao padrão anterior da década de 90. Vale ressaltar o fato de que este aumento recente dos casos pode ser reflexo de projetos de pesquisas realizados recentemente na região leste do estado sobre dinâmica de transmissão da malária autóctone, os quais acabam por detectar mais casos autóctones.

Apesar da tendência decrescente e de números absolutos pequenos, a malária autóctone continua presente no estado de 
São Paulo. As diferenças clínicas e epidemiológicas encontradas neste estudo merecem atenção da vigilância epidemiológica que deve estar preparada e adaptada ao desafio da infecção assintomática ou oligossintomática por Plasmodium ${ }^{14}$. Esta lacuna de conhecimento já foi levantada desde a década de 80 , quando já se apontava a necessidade de estudos sorológicos longitudinais no estado ${ }^{19}$. A busca ativa preventiva talvez seja uma ferramenta a ser novamente utilizada pela vigilância epidemiológica da malária autóctone.

Este estudo visou contribuir para o conhecimento e divulgação da malária autóctone no Estado de São Paulo. Ressalta-se a importância da análise dos dados das fichas de notificação que contém informações fundamentais para a autoavaliação e aprimoramento do sistema de vigilância epidemiológica de malária no estado. Se por um lado a malária deixou de ser uma ameaça para o conjunto da população, por outro passou a ser um problema que afeta grupos restritos em função de seus hábitos ou suas atividades profissionais. É necessária a divulgação destas informações à população sob risco, grupos mais vulneráveis e à categoria médica que, frente à baixa incidência da doença no estado, não faz suspeita clínica precoce. A vigilância epidemiológica do estado atualmente tem os desafios da descentralização do atendimento, diagnóstico e tratamento da malária, lidando com casos mais oligossintomáticos e mantendo o objetivo de manter interrompida a transmissão e de prevenir epidemias em áreas potencialmente transmissoras.

\section{CONFLITO DE INTERESSE}

Os autores declaram não haver nenhum tipo de conflito de interesse.

\section{REFERÊNCIAS}

1. Barata RCB. Malária no Brasil: Panorama Epidemiológico na Última Década. Cad Saúde Pública 1995; 11:128-136.

2. LO SS, Andrade JCR, Condino MLF, Alves MJCP, Semeguini MG, Galvão EC, et al. Malária em usuários de drogas de administração endovenosa associada à soropositividade para HIV. Rev Saúde Pública 1991; 25:17-22.

3. Wanderley DMV, Andrade JCR. Malária induzida no Estado de São Paulo, Brasil. Rev Soc Bras Med Trop 1991; 24:157-161.

4. Barata RCB. Malária e seu controle. São Paulo, SP. Editora Hucitec; 1998. p. 96-97.

5. Secretaria de Vigilância em Saúde, Ministério da Saúde. Brasil. Malária: casos da doença no Brasil. Disponível em: http://www.portal.saude.gov.br/svs/.

6. Secretaria de Vigilância em Saúde, Ministério da Saúde. Brasil. Situação Epidemiológica da Malária no Brasil. Brasília, 2007-2008.

7. Secretaria de Estado de Saúde do Estado de São Paulo (SES-SP/CCD/ CVE). Malária em São Paulo. Boletim Epidemiológico Paulista (BEPA) 2004; 11:10-11.

8. Superintendência de Controle de Endemias do Estado de São Paulo. Vetores e Doenças - Malária. Disponível em: http://www.sucen.sp.gov.br/.

9. Domingos MF, Tubaki RM, Di Santi SM. Malária no Estado de São Paulo: 1975-2006. SUCEN 30 anos. Boletim Epidemiológico Paulista (BEPA) 2006; 3 (S1):10-12.

10. Wanderley DMV, Andrade JCR, Alves MJCP, Alves MCGP, Mattos MR, Gurgel SM, et al. Malária no Estado de São Paulo: avaliação de aspectos da vigilância epidemiológica. Cad Saúde Pública 1989; 5:296-304.

11. Wanderley DMV, Silva RA, Andrade JCR. Aspectos epidemiológicos da malária no estado de São Paulo, Brasil, 1983 a 1992. Rev Saúde Pública 1994; 28:192197.
12. Latorre MRDO, Cardoso MRA. Análise de séries temporais em epidemiologia: uma introdução sobre os aspectos metodológicos. Rev Soc Bras Med Trop 2001; 4:145-152

13. Center for Disease Control and Prevention. Updated guidelines for evaluating public health surveillance systems: recommendations from the guidelines working group. MMWR Morb Mortal Wkly Rep 2001; 50:1-35.

14. Coura JR, Suárez-Mutis M, Ladeia-Andrade S. A new challenge for malaria control in Brazil: asymptomatic Plasmodium infection. Mem Inst Oswaldo Cruz 2006; 101:229-237.

15. Kirchgatter K, Nogueira SL, Padilha A, Curado I, Boulos M, Di Santi SM. Lethal malaria caused by Plasmodium malariae in an asplenic patient in Brazil. BMJ 2005; 331: 576b-b6.

16. Alves MJCP, Mayo RC, Donalisio MR. História, epidemiologia e controle da malária na região de Campinas, Estado de São Paulo, Brasil, 1980 a 2000. Rev Soc Bras Med Trop 2004; 37:41-45.

17. Andrade JCR, Anjos CFD, Wanderley DMV, Alves MJC, Campos PC. Foco de Malária no Estado de São Paulo (Brasil). Rev Saúde Pública 1986; 20:323326.

18. Carvalho ME, Glasser CM, Ciaravolo RMC, Etzel A, Santos LA, Ferreira CS. Nota sobre o encontro de casos autóctones de malária vivax por meio de técnica sorológica, em São Paulo. Cad Saúde Pública 1985; 1:250-252.

19. Carvalho ME, Glasser CM, Santos LA, Ciaravolo RMC. Sorologia de Malária vivax no foco Aldeia dos Índios, Município de Peruíbe, Estado de São Paulo, 1984 a 1986. Cad Saúde Pública 1988; 4:276-292.

20. Carréri-Bruno GC, Ciaravolo RMC, Pereira M. Malária adquirida durante atividade entomológica na Serra do Mar, região Sudeste do Brasil. Rev Saúde Pública 1995; 29: 142-143.

21. Wanderley DMV, Andrade JCR, Meneguetti LC, Chinelatto MJ, Dutra AP. Malária no Estado de São Paulo, Brasil, 1980 a 1983. Rev Saúde Pública 1985; 19: 28-36. 